\title{
Article
}

\section{Fluoxetine induces lipid metabolism abnormalities by acting on the liver in patients and mice with depression}

\author{
Shu-juan PAN², Yun-long TAN², Shang-wu YAO ${ }^{2}$, Yu XIN¹, ${ }^{1,}$ Xuan YANG ${ }^{1,3}$, Jing LIU $^{2}$, Jing XIONG ${ }^{1, *}$ \\ ${ }^{1}$ Department of Pharmacology, Nanjing Medical University, Nanjing 211166, China; ${ }^{2}$ Beijing HuiLongGuan Hospital, Peking University, \\ Beijing 100096, China; ${ }^{3}$ Research Center of Biotechnology, School of Life Science and Technology, China Pharmaceutical University, \\ Nanjing 210009, China
}

\begin{abstract}
Depressive disorders are frequently managed with long-term use of antidepressant medication. Fluoxetine (FLX) is the first selective serotonin reuptake inhibitor to be widely available for the treatment of depression. The present study focuses on the effects and mechanisms of the lipid metabolism abnormalities caused by FLX in patients and in a mouse model of depression. Depression severity was assessed by the Hamilton Depression Scale (HAMD). Triglyceride (TG), cholesterol (TC) and low-density lipoprotein (LDL) serum levels were assessed in 28 patients with depression, aged 31.2 \pm 3.3 years, treated with FLX (20 to 60 mg/day) for 8 weeks. Meanwhile, the serum levels of other lipid metabolism-related parameters, such as high-density lipoprotein (HDL), apolipoprotein A1 (APOA1) and apolipoprotein $\mathrm{B}(\mathrm{ApoB})$, were also determined. The influence of FLX on the hepatic lipid profile and hepatic gene expression of both lipogenic and lipolytic enzymes was evaluated in a mouse model of depression treated with $\mathrm{FLX}\left(10 \mathrm{mg} \cdot \mathrm{kg}^{-1} \cdot \mathrm{d}^{-1}\right.$, ip) for $4 \mathrm{weeks}$. We showed that the serum TG, TC and LDL levels were significantly increased in patients with depression after FLX treatment. The elevation in serum TG levels in the patients was not affected by gender or family history. FLX treatment did not significantly alter serum HDL, APOA1 or APOB levels in the patients. We further demonstrated in mice with depression that FLX treatment increased the hepatic TG level by increasing the expression of lipogenic enzymes and decreasing the expression of lipolytic enzymes in the liver. Antidepressive therapy with FLX is associated with lipid metabolism abnormalities, which are in part mediated by disturbances in hepatic lipid metabolism homeostasis. The findings contribute to the uncovering of metabolic adverse reactions in the pharmacological therapy of depression.
\end{abstract}

Keywords: antidepressant; fluoxetine; metabolic adverse reactions; triglyceride; cholesterol; low-density lipoprotein

Acta Pharmacologica Sinica (2018) 39: 1463-1472; doi: 10.1038/aps.2017.207; published online 10 May 2018

\section{Introduction}

Depression is a highly prevalent, heterogeneous and recurring neuropsychiatric condition characterized by a wide range of symptoms, including altered mood and cognitive functions that lead to serious disabilities and dysfunctions ${ }^{[1]}$. The annual prevalence of depression is reported to be $7 \%$, with a lifetime prevalence of over $15 \%{ }^{[2]}$. Depression has already caused $6 \%$ of the burden of all diseases in Europe regarding disabilityadjusted life years according to WHO data ${ }^{[3]}$. As one of the most prescribed selective serotonin reuptake inhibitors (SSRIs) in the treatment of depression, fluoxetine (FLX) effectively alleviates depressive symptoms with tolerability and safety ${ }^{[4]}$.

\footnotetext{
* To whom correspondence should be addressed.

E-mail xiong.jing@njmu.edu.cn

Received 2017-08-27 Accepted 2017-12-14
}

However, drug adverse reactions including weight gain and dyslipidemia have been reported to be one of the most relevant reasons for the early discontinuation of antidepressant drugs $^{[5]}$. Thus, a better understanding of the effects of FLX on the lipid profile in patients with depression and investigations into the underlying mechanisms are of value to enhance the adherence and tolerance of FLX treatment.

Triglyceride (TG) accumulation in the liver is a frequent indicator of obesity and other related metabolic disorders ${ }^{[5,6]}$. Fatty acid and TG synthesis in the liver is a finely tuned metabolic pathway that is essential for the production of low-density lipoprotein (LDL), which is used for TG and cholesterol (TC) exportation into the plasma and delivery to other tissues $^{[7]}$. Therefore, lipid homeostasis can be achieved by modulation of the expression of both lipogenesis- and lipolysisrelated enzymes ${ }^{[8]}$. Fatty acid synthase (FAS) and acetyl- 
CoA carboxylase (ACC) are important enzymes that regulate the process of fatty acid and TG synthesis ${ }^{[9]}$. Meanwhile, carboxylesterases are the most important liver lipases, which catalyze TG degradation in the hepatocytes and contribute to hepatic TG metabolism ${ }^{[10]}$. Mouse carboxylesterase (Ces) 1d, also known as TG hydrolyase, is an ortholog of human CES1. Ces1d is highly expressed in the liver and plays a critical role in hepatic TG mobilization and thus regulates TG storage ${ }^{[11]}$. Mouse Ces1e (esterase-x), also highly expressed in the liver, is a close homolog of human CES2. The conserved esterase/ lipase active site motif in Ces1e plays a similar role as Ces1d in lowering hepatic TG levels. In addition, Ces1e also prevents hepatic TG accumulation by increasing the redirection to $\beta$-oxidation ${ }^{[12]}$.

In our previous study using primary cultured mouse hepatocytes, FLX was demonstrated to induce lipid accumulation by suppressing AMP-activated protein kinase (AMPK), subsequently increasing the expression of ACC and FAS and decreasing the expression of Ces1d and Ces1e. However, whether the findings in vitro are comparable with lipid metabolism alterations in FLX-administered patients with depression is not clear. To further investigate the role of the liver in FLX-induced lipid metabolism abnormalities, the lipid profile was observed in depressive patients given FLX treatment, while a mouse model with depression was established, and lipid accumulation as well as the expression of lipogenic and lipolytic genes in the mouse liver tissues were evaluated. The present findings are of importance to the in-depth understanding of FLX-induced lipid metabolism abnormalities during the medical treatment of patients with depression.

\section{Methods and materials}

\section{Subjects and clinical study design}

Twenty-eight newly admitted inpatients (male/female=8/20) were recruited for the study in the Beijing HuiLongGuan Hospital, China during January 2013 to October 2016. All patients met the following inclusion criteria: (1) Han Chinese, 20-65 years of age; (2) depressed subjects diagnosed with MDD (major depressive disorder) without psychotic features, according to the Structured Clinical Interview for DSM-IV $(\mathrm{SCID})^{[13]}$, with a current 17 -item Hamilton Depression Scale (HAMD) rating of $\geq 15$. All diagnoses were confirmed by clinical interview with a board-certified psychiatrist. Depressed patients were excluded for the presence of the following: a history of schizophrenia (determined by SCID), bipolar disorder (determined by SCID), or any eating disorder or post-traumatic stress disorder (PTSD) within one month or substance abuse within six months (determined by SCID). Further, study participants had no acute illnesses or infections, chronic inflammatory disorders, neurological disorders, or any other major medical conditions considered to be potentially confounding (eg, diabetes, HIV, cancer, history of cardiovascular disease, etc) (determined by physical examination and laboratory testing). All subjects provided written informed consent. The study did not interfere with the treatment of the patients and was approved by the Institutional Review Board of Bei- jing HuiLongGuan Hospital.

\section{Data on demographic characteristics}

The clinical data retrieved included age, nationality, sex, marital status, body mass index (BMI) and family history. Hamilton Depression Scale (HAMD) scores were routinely collected in Beijing HuiLongGuan Hospital. HAMD scores were determined by the patients' attending psychiatrist following an interview conducted within $48 \mathrm{~h}$ after admission and again 8 weeks later as a routine practice of the inpatient unit. HAMD scores were used as a measure of depression. The demographic and clinical characteristics of the sample are presented in Table 1.

\section{Animals and reagents}

Male C57BL/ 6 mice, aged 8 weeks at the beginning of the CMS, were maintained in a specific pathogen-free environment in Nanjing Medical University. All protocols were approved by the Institutional Animal Care and Use Committee of Nanjing Medical University and in strict accordance with the National Institutes of Health Guide for the Care and Use of Laboratory Animals. The TG and TC serum levels were measured using a kit from Beckman Coulter Company (Fullerton, CA, USA), while TG and TC level in liver tissues were determined using a kit from Applygen Technologies Inc (Beijing, China). FLX was purchased from Sigma (St Louis, MO, USA). Polyvinylidene difluoride membranes were from Bio-Rad Laboratories (Hercules, CA, USA). Mouse Ces1d and Ces1e were detected by antibodies against human CES1 and human CES2, respectively, as reported previously ${ }^{[14-16]}$. Other primary antibodies used in this study were as follows: antiACC1 (Abcam, Cambridge, UK), anti-FAS, and anti-GAPDH, and anti- $\beta$-actin (Bioworld, St Louis Park, USA). The goat anti-rabbit IgGs conjugated with horseradish peroxidase were from Pierce Chemical (Pierce, Rockford, IL, USA). All other reagents were of analytical grade and commercially available.

\section{CMS procedure}

Mice were singly housed, allowed to acclimate for 1 week and were then subjected to 8 weeks of stressors, including inversion of the day/night light cycle, wet cage bedding, tilted cages, restraint, forced swim stress (for $6 \mathrm{~min}$ ), overnight food and water deprivation, and paired housing. These stressors were mild and unpredictable in nature, duration and frequency. Control mice were held in another room and were group-housed ( $n=4 /$ cage). The animals' body weights were evaluated every week. Mice treated with CMS for 4 weeks began to be treated with fluoxetine $\left(10 \mathrm{mg} \mathrm{kg}^{-1} \cdot \mathrm{d}^{-1}\right.$, ip) for 4 weeks when depressive behaviors occurred. The dose of FLX was chosen based on our preliminary study and published studies $^{[17-19]}$. The emotional state of the animals was evaluated by the sucrose preference test, tail suspension test (TST) and forced swim test (FST).

\section{Sucrose preference test}

Mice were first habituated to drink from two bottles with 
Table 1. Demographic, clinical characteristics and metabolic indicators of patients with depression in the pre-treatment and post-treatment of fluoxetine.

\begin{tabular}{|c|c|c|c|c|}
\hline Demographic specialties & & Pre-treatment & Post-treatment & Statistical evaluation \\
\hline Age (mean $\pm S D)$ & & $31 \pm 13$ & NA & NA \\
\hline Nationality & Han Chinese & $28(100 \%)$ & NA & NA \\
\hline Sex & Male & $8(28.6 \%)$ & & \\
\hline Marital status & Single & $16(57.1 \%)$ & NA & NA \\
\hline Family history & Negative & $21(75.0 \%)$ & & \\
\hline HAMD & & $37.5 \pm 20.8$ & $10.8 \pm 7.4$ & $Z=-4.625 ; P<0.001$ \\
\hline $\operatorname{BMI}\left(\mathrm{kg} / \mathrm{m}^{2}\right)$ & & $23.3 \pm 2.3$ & $23.2 \pm 2.1$ & $Z=-0.834 ; P=0.404$ \\
\hline $\mathrm{TG}(\mathrm{mmol} / \mathrm{L})$ & & $1.09 \pm 0.70$ & $1.82 \pm 0.85$ & $Z=4.623 ; P<0.001$ \\
\hline TC (mmol/L) & & $4.37 \pm 0.82$ & $5.2 \pm 1.06$ & $Z=4.190 ; P<0.001$ \\
\hline LDL (mmol/L) & & $1.44 \pm 0.37$ & $1.45 \pm 0.34$ & $Z=2.141 ; P=0.032$ \\
\hline Insulin $(\mu \mid \mathrm{U} / \mathrm{mL})$ & & $7.0 \pm 3.7$ & $7.1 \pm 3.5$ & $Z=0.512 ; P=0.608$ \\
\hline
\end{tabular}

All demographic data were reported as mean \pm SD or rate (\%). Clinical characteristics and metabolic indicators were analyzed using the Wilcoxon $t$ tests.

one containing a sucrose solution $(1 \%)$ and the other containing only tap water. The side of the bottles in the cage were switched each day to prevent possible effects of side preference in drinking behavior. Before the test, all mice were deprived of water and food for $12 \mathrm{~h}$. The intake of tap water and sucrose solution was recorded simultaneously by weighing the bottles. The sucrose preference was measured as a percentage of the consumed sucrose solution relative to the total amount of liquid intake.

\section{Tail suspension test}

Mice were individually suspended by wrapping their tail with tape and fixed upside down on the hook. Immobility time (in seconds) was automatically recorded over a 6-min test period. Immobility was considered only when the mice hung passively and completely motionless. The immobility time of the mice in the last 4 min was analyzed with an automated TailSuspScan apparatus (Clever Sys).

\section{Forced swim test}

Mice were forced to swim in a glass barrel (diameter, 15 $\mathrm{cm}$; height, $25 \mathrm{~cm}$ ) with $14 \mathrm{~cm}$ of water at room temperature (approximately $22 \pm 1^{\circ} \mathrm{C}$ ). A mouse was placed in the barrel, and immobility behavior was observed for $6 \mathrm{~min}$ when the mouse floated in the water without struggling, making only small movements necessary to keep its head above the water. The duration of immobility was recorded in the last 4 min of the 6-min period by an automated TailSuspScan apparatus (Clever Sys). The immobility behavior observed in the test was considered to reflect depressive-like symptoms.

\section{Measurement of TG and TC levels in the serum}

Venous blood was collected into tubes containing coagulation accelerator thrombin and centrifuged at 4000 revolutions per minute for $5 \mathrm{~min}$. Serum samples from patients were collected between 7 and 9 AM before and after 8 weeks of FLX treatment. Serum TG, TC, LDL, HDL, APOA1, APOB, glucose and insulin levels were determined using a kit in an autoanalyzer (AU5800; Beckman Coulter Inc, Fullerton, CA, USA). Lab personnel who performed the assay were blind to the demographic and clinical data of the patients. The reference ranges were as follows: $0.00-1.70 \mathrm{mmol} / \mathrm{L}$ for serum TG levels, 1.00-5.17 mmol/L for serum TC levels, $1.90-3.60 \mathrm{mmol} / \mathrm{L}$ for serum LDL levels, $0.71-1.68 \mathrm{mmol} / \mathrm{L}$ for serum HDL levels, 1.00-2.60 mmol/L for serum APOA1 levels, 0.50-1.10 mmol/L for serum APOB levels, 3.9-6.1 mmol/L for fasting glucose levels and 1.9-23 $\mathrm{IIU} / \mathrm{mL}$ for insulin levels.

\section{H\&E staining}

Specimens from mouse liver tissues were fixed in 10\% neutral buffered formalin, paraffin-embedded, cut into 4 - $\mu \mathrm{m}$ serial sections, and stained with hematoxylin and eosin (H\&E) for histological examination. Morphometric analysis was carried out using a Leica DM6000B light microscope (Leica Microsystems, Wetzlar, Germany).

\section{Preparation of S9 fractions}

The frozen liver tissues were thawed in homogenization buffer $(50 \mathrm{mmol} / \mathrm{L}$ Tris- $\mathrm{HCl}, \mathrm{pH} 7.4,150 \mathrm{mmol} / \mathrm{L} \mathrm{KCl}$ and 2 mmol/L EDTA) and then homogenized on ice with 6 passes of a Teflon pestle driven by a Wharton stirrer. The homogenates were centrifuged at $10000 \times \mathrm{g}$ for $20 \mathrm{~min}$ at $4{ }^{\circ} \mathrm{C}$. The S9 frac- 
tions of the liver tissues (supernatant) were assayed using a Western blot assay.

\section{Western blot analysis}

Protein concentrations were determined with a BCA protein assay based on the albumin standard (Pears, Rockford, IL, USA). Equal amounts of protein were separated on a $10 \%$ SDS-PAGE and transferred electrophoretically onto PVDF membranes. The membranes were blocked with 5\% non-fat milk in Tris-buffered saline/0.1\% Tween 20 for $2 \mathrm{~h}$, subsequently blotted with the respective primary antibodies overnight, and then blotted with horseradish peroxidaseconjugated secondary antibody for $1 \mathrm{~h}$. The protein bands were visualized with an enhanced chemiluminescence detection system. Protein levels were quantified by density analysis using ImageJ software (NIH) and expressed as the protein of interest/internal control.

\section{Statistical analysis}

Data are expressed as the mean \pm SD. Statistical analysis was performed using SPSS 17.0 (Chicago, IL, USA). The Wilcoxon $\mathrm{T}$ test was used for assessing changes in the values of metabolic parameters and the HAMD scores before and after FLX treatment. Differences between FLX-treated mice and vehicletreated mice were tested with two-way ANOVA, followed by Bonferroni post hoc test. $P<0.05$ was considered statistically significant.

\section{Results}

Participants and clinical characteristics of depression patients pretreatment and posttreatment with FLX

Twenty-eight patients with depression including 8 males and 20 females (mean age $\pm S D=31.2 \pm 13.3$ years) received medical treatment with FLX (20 to $60 \mathrm{mg} /$ day) for 8 weeks. The baseline clinical characteristics of patients are shown in Table 1. As shown in Table 1, the patients with depression had obvious psychopathological symptoms, with an average HAMD score of 37.5 \pm 20.8 . After 8 weeks of medical treatment, their average HAMD score was reduced to $10.8 \pm 7.4(P<0.001, Z=-4.625)$.

Serum TG and TC levels were higher in patients with depression after FLX treatment for 8 weeks in comparison to the levels pretreatment $(1.09 \pm 0.70 \mathrm{mmol} / \mathrm{L}$ vs $1.82 \pm 0.85$ $\mathrm{mmol} / \mathrm{L}$ and $4.38 \pm 0.82 \mathrm{mmol} / \mathrm{L}$ vs $5.20 \pm 1.06 \mathrm{mmol} / \mathrm{L}$, respectively) $(P<0.001)$. All patients showed increased serum TG levels, and $96.4 \%$ of patients showed increased serum TC levels. The average increase was $39.9 \%$ for serum TG and $14.4 \%$ for serum TC after FLX treatment. Serum LDL levels were also significantly higher in patients with depression after FLX treatment for 8 weeks than levels pretreatment $(1.44 \pm 0.37 \mathrm{mmol} / \mathrm{L}$ vs $1.45 \pm 0.34 \mathrm{mmol} / \mathrm{L})(P<0.05)$. The elevation in serum TG and TC levels after FLX treatment was consistent with the decrease in HAMD scores of the patients (Table 1). As other lipid metabolism-related indicators, levels of serum HDL, APOA1, APOB, fasting glucose and insulin in the peripheral blood were also measured. FLX treatment for 8 weeks did not significantly alter the levels of serum HDL, APOA, APOB, glucose or insulin $(2.44 \pm 0.70 \mathrm{mmol} / \mathrm{L}$ vs $2.71 \pm 0.87 \mathrm{mmol} / \mathrm{L}$, $2.00 \pm 0.39 \mathrm{mmol} / \mathrm{L}$ vs $2.06 \pm 0.39 \mathrm{mmol} / \mathrm{L}, 0.94 \pm 0.40 \mathrm{mmol} / \mathrm{L}$ vs $0.94 \pm 0.39 \mathrm{mmol} / \mathrm{L}, 4.95 \pm 0.7 \mathrm{mmol} / \mathrm{L}$ vs $4.93 \pm 0.5 \mathrm{mmol} / \mathrm{L}$, and $7.0 \pm 3.7 \mu \mathrm{IU} / \mathrm{mL}$ vs $7.1 \pm 3.5 \mu \mathrm{IU} / \mathrm{mL}$, respectively) $(P>0.05)$. Meanwhile, the BMI of the patients was also not affected by FLX treatment $(23.3 \pm 2.3$ vs $23.2 \pm 2.1)(P>0.05)$ (Table 1).

The influence of gender and family history on serum TG and TC levels was further investigated. Both female and male patients with depression had higher serum TG levels after FLX treatment for 8 weeks than before treatment (Female: $P<0.001$, $Z=3.920$; Male: $P<0.001, Z=2.521$ ) (Figure $1 \mathrm{~A}$ ). Serum TG levels were significantly higher in male patients than female patients (pretreatment: $P=0.005, Z=-2.747$; posttreatment: $P=0.016, Z=-2.390)$. Similarly, female patients with depression had higher serum TC levels after FLX treatment for 8 weeks than prior to treatment $(P<0.001, Z=2.521)$, while the difference in serum TC levels in male patients between pretreatment and posttreatment was not statistically significant $(P=0.123$, $\mathrm{Z}=1.540$ ) (Figure 1B). In contrast to TG levels, the difference in serum TC levels between female and male patients was not statistically significant (pretreatment: $P=0.304, Z=-1.043$; posttreatment: $P=0.409, \mathrm{Z}=-0.865)$.

Furthermore, both family history-positive and -negative patients with depression had higher serum TG and TC levels after 8 weeks of FLX treatment than pretreatment (Positive: $P=0.018, Z=2.366$; Negative: $P=0.001, Z=3.435$ ) (Figure 2). No
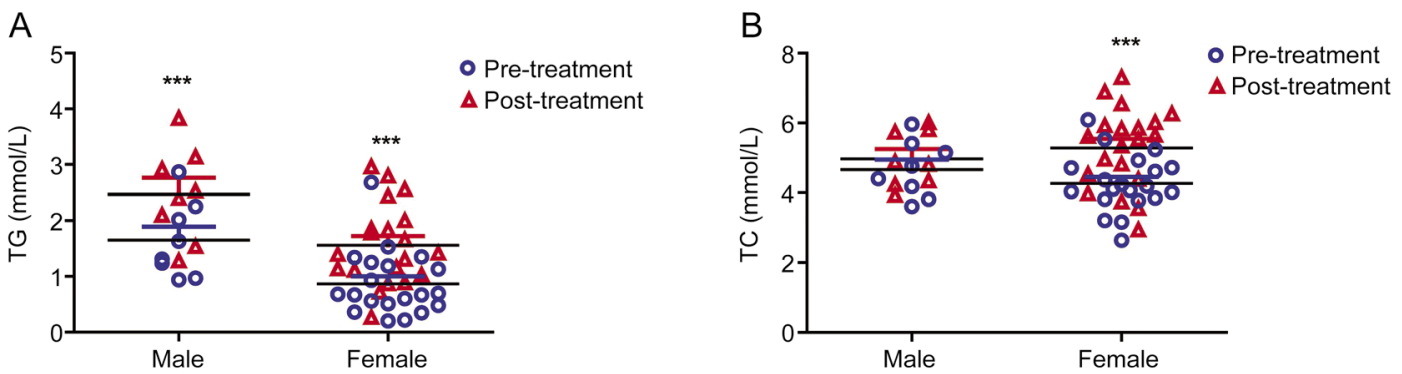

Figure 1. Gender differences in serum TG and TC levels. Serum TG levels were elevated in both male and female MDD patients after FLX treatment compared to the levels prior to treatment $(P<0.001)$, while serum TC levels were elevated in female MDD patients after FLX treatment $(P<0.001)$ but not significantly different in male patients $(P>0.05)$. Data are expressed as the mean \pm SD. ${ }^{* * *} P<0.001$ vs prior to FLX treatment (TG: Male: $P<0.001$, $Z=2.521$, Female: $P<0.001, Z=3.920$; TC: Female: $P<0.001, Z=2.521)$. 

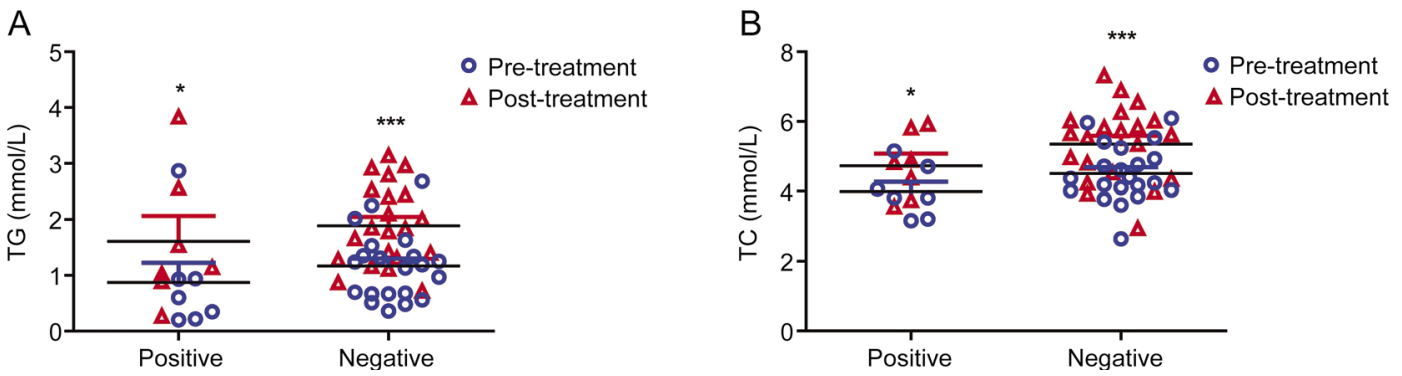

Figure 2. Family history differences in serum TG and TC levels. Serum TG and TC levels were elevated after FLX treatment in MDD patients with positive and negative family history $(P<0.001)$. No statistically significant difference in serum TC levels existed between family history-positive and -negative patients $(P>0.05)$. ${ }^{*} P<0.05,{ }^{* * *} P<0.001$ vs prior to FLX treatment (TG: Positive: $P=0.018, Z=2.366$, Negative: $P<0.001, Z=4.015$; TC: $P$ ositive: $P=0.018$, $Z=2.366$, Negative: $P<0.001, Z=3.528$ ).

statistically significant difference in serum TG or TC levels existed between family history-positive and -negative patients (pretreatment: $P=0.090, Z=1.725$ for TG; $P=0.113, Z=1.618$ for TC; posttreatment: $P=0.296, Z=1.088$ for TG; $P=0.208, Z=1.300$ for TC).

Triglyceride levels in the liver tissues are elevated by FLX in mice with depression

To further demonstrate the effects and mechanisms of FLX on the lipid metabolism abnormalities, mice with depression were prepared by exposure to chronic mild stress for 4 weeks, and then FLX was administered simultaneously for another 4 weeks once depressive behaviors were exhibited. FLX treatment significantly increased sucrose preference (Figure 3A) and decreased the immobility duration in the TST and FST compared to non-treatment (Figure 3B and 3C), which indicated an improvement in depressive behaviors. Meanwhile, the liver tissues were dissected, and the TG and TC levels in the liver tissues were determined. FLX treatment significantly increased hepatic TG levels in both control and depressive mice by 1.47 -fold and 1.39 -fold, respectively, compared to non-treatment (Figure 4A), while the hepatic TC levels were not significantly altered by FLX treatment (Figure 4B). Furthermore, a routine histological examination was performed. Lipid droplets in the liver were shown to be larger and more numerous in both control and depressive mice given FLX than in those not treated with FLX as revealed by H\&E staining (Figure 5). The data implied that lipids, especially TGs, were accumulated in the liver in depressive mice treated with FLX, which was consistent with the above clinical data and data
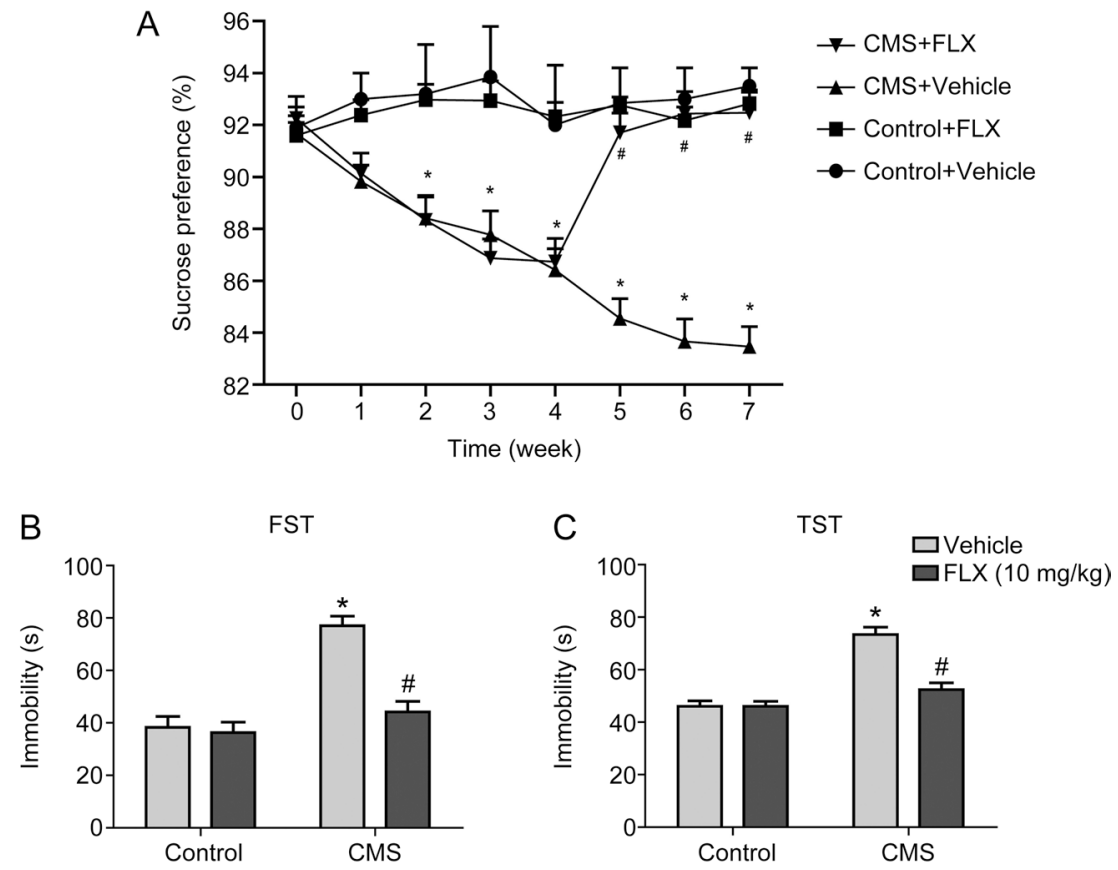

Figure 3. FLX improves the depressive behaviors in mice with depression. Mice were treated with mild stressors that were unpredictable in nature, duration and frequency. $\mathrm{FLX}\left(10 \mathrm{mg} \cdot \mathrm{kg}^{-1} \cdot \mathrm{d}^{-1}\right)$ was administered intraperitoneally for 4 weeks once depressive behaviors occurred. (A) Sucrose preference. (B) Tail suspension test. (C) Forced swim test. ${ }^{*} P<0.05$ vs control mice, ${ }^{\#} P<0.05$ vs mice without FLX treatment ( $n=6 /$ group). 

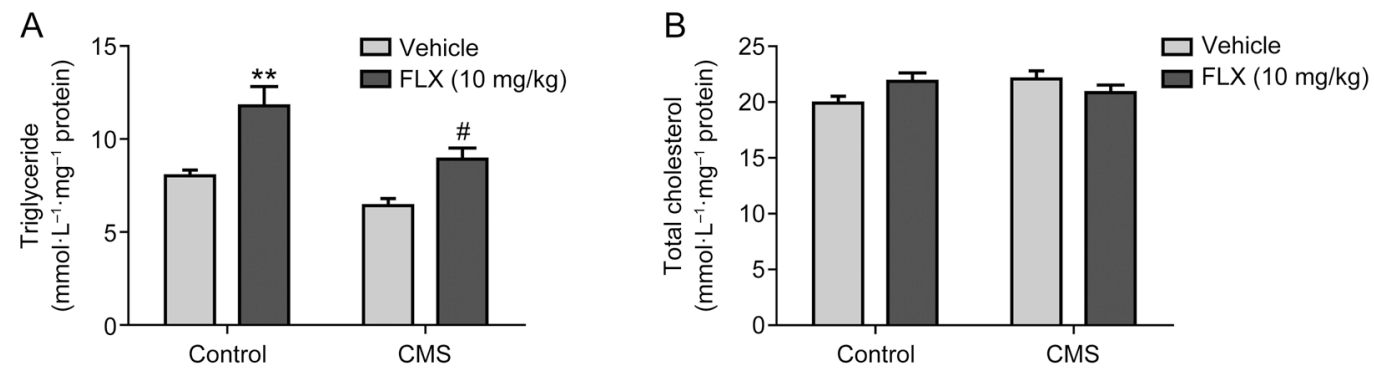

Figure 4. Effects of FLX on the hepatic $T G(A)$ and $T C$ (B) levels in the liver tissues of mice with depression. Mice livers were dissected, and subsequently, the TG and TC in the liver tissues were extracted and measured using commercial kits. ${ }^{* *} P<0.01$ vs control mice, ${ }^{\#} P<0.05$ vs mice with depression ( $n=6 /$ group).

obtained previously in vitro ${ }^{[14]}$.

FLX regulates the expression of lipogenic and lipolytic genes in the liver tissues of both control mice and mice with depression

Next, the expression of lipogenic genes, FAS and ACC, and lipolytic genes, Ces1d and Ces1e, in the liver of mice was investigated using Western blot analysis. With FLX treatment, the expression levels of FAS and ACC were increased by 1.23and 1.39-fold, respectively, in the liver tissues of control mice and by 1.39- and 1.55-fold in the liver of mice with depression compared to the levels in the respective non-treated mice (Figure 6A-6C). In contrast, the expression levels of Ces1d and Ces1e in the livers of FLX-treated control and depressed mice were significantly decreased to $71 \%$ and $52 \%$ of those in the liver of non-treated control mice and to $84 \%$ and $62 \%$ of those in the liver of non-treated depressed mice, respectively (Figure 6A, 6D and 6E). The results con- firmed that the effects of FLX on the lipid profile and hepatic lipid accumulation were at least in part imposed by directly acting on the liver.

\section{Discussion}

SSRIs are a cornerstone in the pharmacological treatment of depression, with fewer anti-cholinergic side effects, less toxicity and better tolerance for the patients than previously used treatments ${ }^{[20]}$. However, SSRI treatment has been associated with adverse reactions such as weight gain, sexual dysfunction, drug interactions and extrapyramidal symptoms ${ }^{[21,22]}$. The adverse reactions have become one of the leading causes of unexpected early discontinuation of SSRI medical treatment. FLX was the first SSRI developed and has since become the most widely prescribed antidepressant ${ }^{[23]}$. Therefore, the present study aimed to understand the effects and mechanisms of the metabolic adverse effects caused by FLX in order
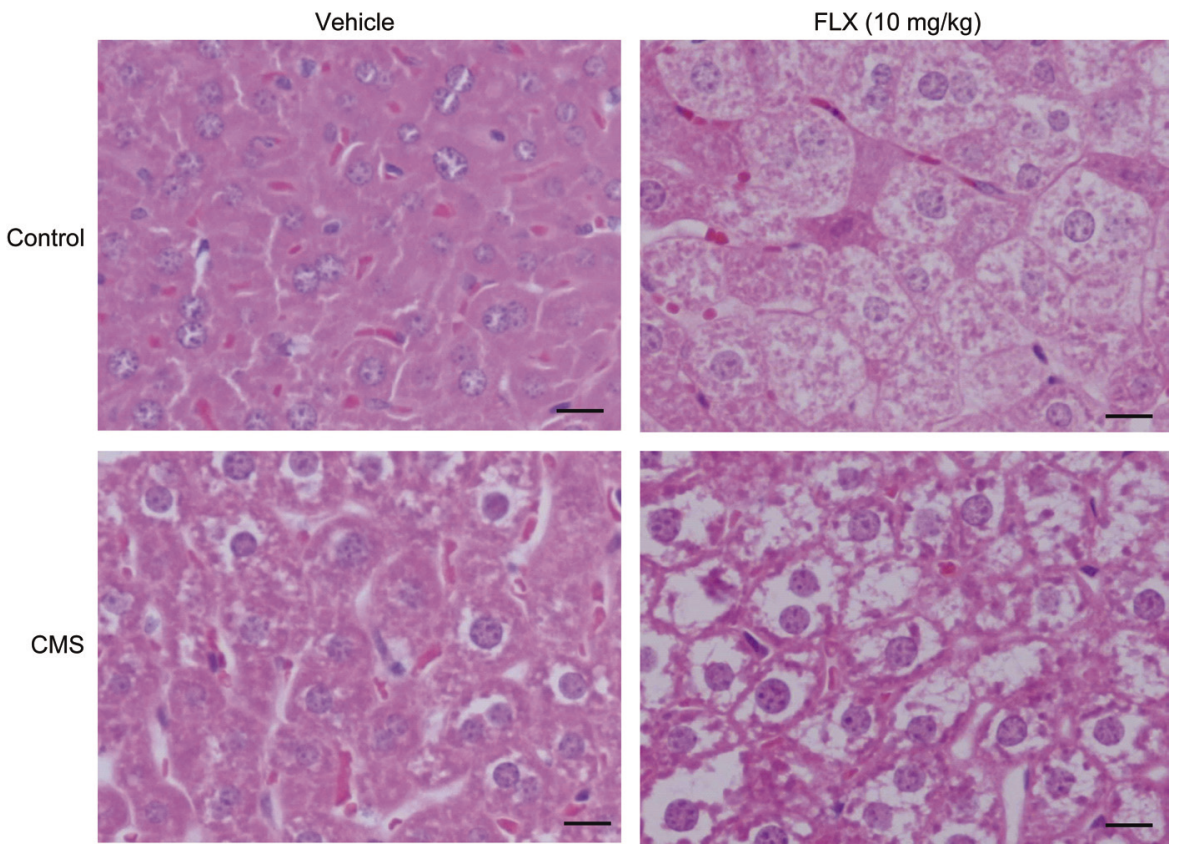

Figure 5. Effects of FLX on the hepatic lipid accumulation in the liver tissues of mice with depression. Serial sections of the mouse liver were stained with H\&E for routine histological examination. Images shown in this figure (200x) were representative of the liver sections from six mice. 

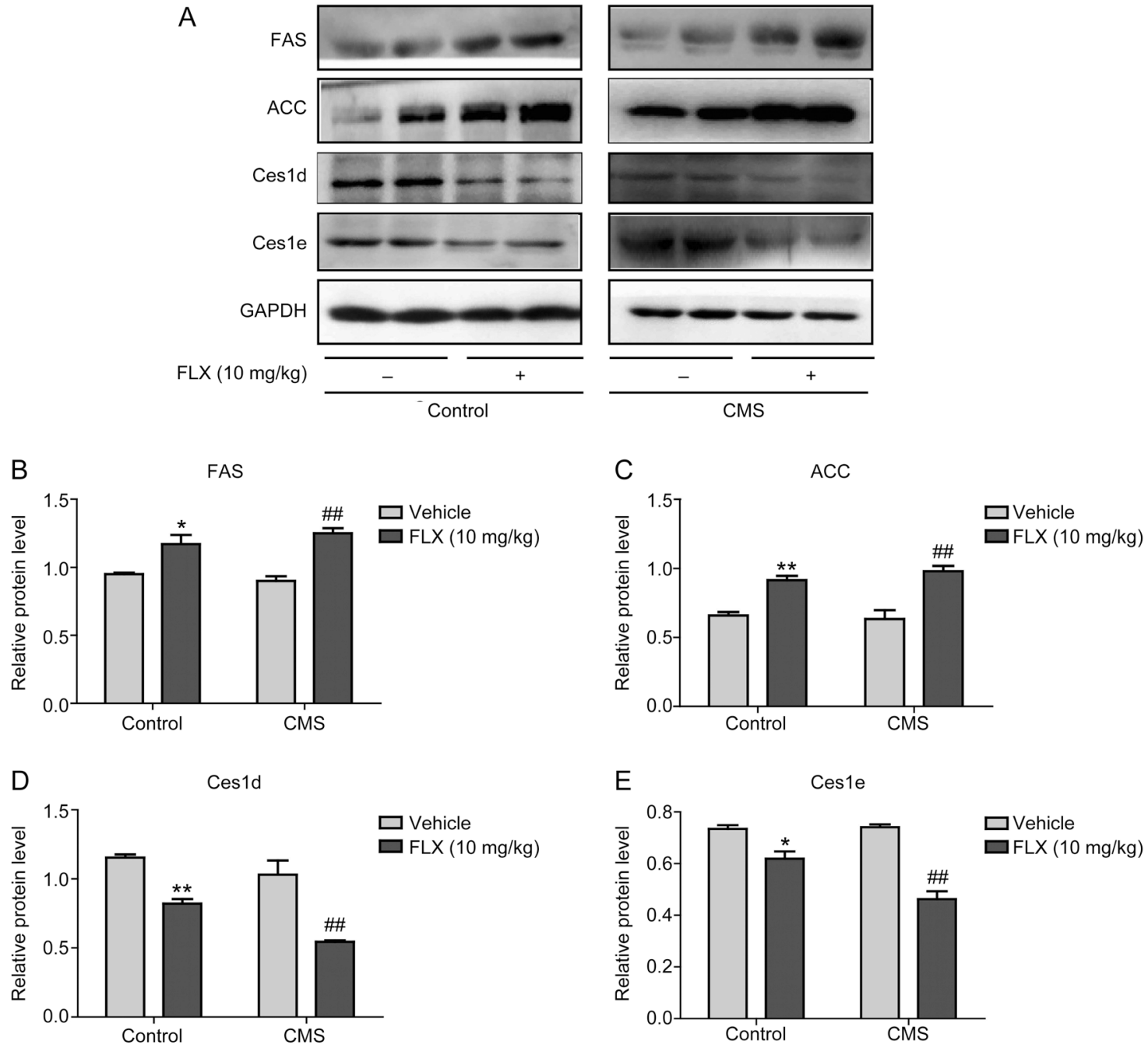

Figure 6. Effects of FLX on the expression of hepatic lipogenic and lipolytic genes in the liver tissues of mice with depression. Liver S9 fractions were analyzed for the expression of ACC1, FAS, CES1 and CES2 using Western blot analysis. ${ }^{*} P<0.05,{ }^{* *} P<0.01$ vs control mice, ${ }^{\# \#} P<0.01$ vs mice with depression ( $n=6$ /group). The protein bands from the Western blot films were scanned and quantified using ImageJ (NIH) and then normalized to the internal control. Two-way ANOVA was used to analyze the differences between FLX-treated mice and vehicle-treated mice, followed by Bonferroni post hoc test.

to provide potential drug targets to alleviate those adverse effects.

HAMD-17 scores, as an indicator of depression in patients, include six items: anxiety (psychic), anxiety (somatic), somatic symptoms (gastrointestinal), somatic symptoms (general), hypochondriasis and insight ${ }^{[24]}$. The primary FLX efficacy measure for this study was the HAM-D-17 total scores, for which a decrease is indicative of an improvement in the symptoms of depression ${ }^{[25]}$. Before medical treatment with FLX, the HAMD-17 scores were markedly higher in patients with depression than the scores after treatment, and the HAMD-17 scores decreased significantly after 8 weeks of FLX treatment in our study (Table 1).

The present study demonstrated that 8 weeks of FLX treatment resulted in an increase in serum TG, TC and LDL levels in patients with depression, along with a decrease in the HAMD-17 score (Table 1). Our findings are consistent with the results of a previous study that reported that FLX treatment was associated with abdominal obesity and hypercholesterolemia ${ }^{[26]}$. A number of studies have demonstrated that SSRIs induce an increase in serum TG, TC and LDL levels in patients with psychiatric disorders. A significant association between SSRI serum concentrations and the TG, TC and LDL cholesterol levels, as well as the risk of metabolic syndrome, has been demonstrated ${ }^{[27]}$. Treatment with the SSRI paroxetine resulted in an average individual increase in LDL cholesterol levels of $9 \%$ in both male healthy control subjects and patients with panic disorder, and the increases in LDL cholesterol were normalized after discontinuation of therapy ${ }^{[28]}$. Younger subjects showed a higher increase in LDL cholesterol at the treatment phase than older subjects ${ }^{[28]}$. A double-blind, randomized, placebo-controlled efficacy clinical study comparing the efficacy of short-term administration of paroxetine, venlafaxine and placebo in patients with social anxiety disorder 
determined that paroxetine and venlafaxine, a serotonin and norepinephrine reuptake inhibitor, caused a statistically significant increase in total cholesterol, but the rise in LDL cholesterol did not reach statistical significance ${ }^{[29]}$. In the present study, the data showed that the elevation in serum TG levels was not affected by gender or family history, while the elevation in serum TC levels was not affected by family history but was affected by gender (Figure 1 and 2). There was no significant difference in serum TC levels pre- and posttreatment with FLX in male patients. The possible reason for the differences between male and female patients could be due to differences in age, in which the average age was approximately 40 years old for the male patients and approximately 25 years old for the female patients. Younger subjects have previously been reported to have lower baseline TC levels, while lipid levels increase with age ${ }^{[30]}$, which provides a possible explanation for the gender differences in the serum TC levels in this research subject population. Notably, no significant alteration in serum TC levels was observed in the mice treated with FLX for 4 weeks (Figure 4B), since male mice are usually used in establishing mouse models of depression to avoid gender differences in in vivo studies ${ }^{[18]}$. The animal results in Figure $4 \mathrm{~B}$ are consistent with the gender differences in the serum TC levels found in the clinical subjects (Figure 1B). The gender differences in the effects of antidepressants on cholesterol metabolism in vivo deserve further elucidation in future studies. Meanwhile, other lipid metabolism-related indicators were detected, and no alteration was found in the serum levels of HDL, APOA1, APOB, glucose or insulin after FLX treatment.

However, our results are in conflict with several previous studies that found significant reductions in serum TG and TC levels, as well as a significant increase in HDL cholesterol, with FLX therapy in mixed populations of obese individuals $^{[31-33]}$. A significant decrease in serum TG and TC levels after 4 weeks of FLX treatment and a non-statistically significant decrease after 8 weeks of treatment have been reported in patients with depression ${ }^{[34]}$. Another recent study also reported that treatment with antidepressants (sertraline, escitalopram, fluoxetine, and venlafaxine) for 8 weeks increased the serum TC and HDL levels of the outpatients ${ }^{[35]}$. Meanwhile, some studies support our findings, as no differences in LDL or HDL levels were observed between patients with and without antidepressants and healthy controls ${ }^{[36]}$, and 12 weeks of sertraline treatment failed to significantly alter BMI, body weight, glucose, HDL-C or LDL-C ${ }^{[37]}$. The contrasting findings are most likely due to differences in methodologies or patient populations or problems in the sample sizes. This controversial question deserves further elucidation by exploration of the in-depth mechanisms.

The underlying mechanism by which FLX treatment increases serum TG, TC, and LDL levels in patients with depression needs to be clarified. In a previous study, a cell biology experiment demonstrated that FLX promoted lipid accumulation in primary cultured hepatocytes. The causes of hepatic lipid metabolism disorders induced by FLX are related to increases in TG synthesis and decreases in TG deg- radation $^{[14,15]}$. Therefore, we focused on liver-centered lipid metabolism disorders in the model mice with depression in the present study in order to provide a compatible explanation for the increase in serum TG, TC and LDL levels after FLX treatment in the clinical trial. While the body weight of the mice is not altered significantly by the treatment of FLX (data not shown), the increased TG accumulation is observed in the liver of both control and model mice with FLX treatment (Figure 4 and 5). The following results further implied that the increase in liver lipogenesis and decrease in liver lipolysis are involved in the FLX-induced alteration in the lipid profile in patients with depression (Figure 6). This is the first study to demonstrate that antidepressant drugs alter the hepatic lipid profile and expression of lipid metabolizing enzymes in animals with depression, although the administration of olanzapine has been reported to increase body weight and food intake in healthy control animals ${ }^{[38]}$.

The major limitation of our study was the limited number of patients with depression, which did not allow for subclassification based on the treatment response. Such a study design would be of particular interest to further interpret the underlying mechanism of side effects associated with pharmacological therapy in the treatment of depression. Furthermore, a larger study cohort including larger subgroups such as malesfemales, smokers-nonsmokers would have better demonstrated the effect of confounding factors.

The present study highlights three important findings. First, there was a trend toward a side effect of an increase in serum TG, TC and LDL levels induced by FLX, which will likely guide the safer use of clinical medications. Second, FLX altered the hepatic lipid profile in mice with depression, significantly elevating the level of TG in liver tissues. Third, increased expression of lipogenic enzymes and decreased expression of lipolytic enzymes were shown to be involved in the disorders of lipid metabolism induced by FLX treatment. These findings are important for unveiling an in-depth understanding of the metabolic adverse reactions related to antidepressant pharmacological therapy and suggesting potential drug targets to interfere with those adverse reactions.

\section{Acknowledgements}

We would like to thank all of the study participants and staff. This study was supported by the National Natural Science Foundation of China (No 81302855), the Natural Science Foundation of Jiangsu Province, China (№ BK2012446), Beijing Municipal Administration of Hospitals Clinical Medicine Development of Special Funding Support (XMLX201513).

\section{Author contribution}

Shu-juan PAN conducted the literature review, data analysis, data collection and manuscript preparation; Yun-long TAN was involved in the data analysis and manuscript preparation; Shang-wu YAO was involved in the data collection and data analysis; Yu XIN was involved in the molecular biology experiments; Xuan YANG was involved in the animal experiments; Jing LIU was involved in the data collection and data 
analysis; Jing XIONG conducted the molecular experiments, literature review, data analysis, data interpretation and manuscript preparation.

\section{References}

1 Zunszain PA, Hepgul N, Pariante CM. Inflammation and depression. Curr Top Behav Neurosci 2013; 14: 135-51.

2 DelMastro K, Hellem T, Kim N, Kondo D, Sung YH, Renshaw PF. Incidence of major depressive episode correlates with elevation of substate region of residence. J Affect Disord 2011; 129: 376-9.

3 Sobocki P, Jonsson B, Angst J, Rehnberg C. Cost of depression in Europe. J Ment Health Policy Econ 2006; 9: 87-98.

4 Garrison GD, Levin GM. Factors affecting prescribing of the newer antidepressants. Ann Pharmacother 2000; 34: 10-4.

5 Nassir F, Adewole OL, Brunt EM, Abumrad NA. CD36 deletion reduces VLDL secretion, modulates liver prostaglandins, and exacerbates hepatic steatosis in ob/ob mice. J Lipid Res 2013; 54: 2988-97.

6 Richard J, Lingvay I. Hepatic steatosis and Type 2 diabetes: current and future treatment considerations. Expert Rev Cardiovasc Ther 2011; 9: 321-8.

7 Estridge TB, Dey AB, Reidy C, Yu X, Zhang Y, Hartley M, et al. Identification of 4-aminopyrazolopyrimidine metabolite that may contribute to the hypolipidemic effects of LY2584702 in long evans diet-induced obese rats. J Pharmacol Exp Ther 2017; 362: 108-18.

8 Horton JD, Goldstein JL, Brown MS. SREBPs: activators of the complete program of cholesterol and fatty acid synthesis in the liver. J Clin Invest 2002; 109: 1125-31.

9 Tang X, Li J, Xiang W, Cui Y, Xie B, Wang X, et al. Metformin increases hepatic leptin receptor and decreases steatosis in mice. J Endocrinol 2016; 230: 227-37.

10 Quiroga AD, Lehner R. Role of endoplasmic reticulum neutral lipid hydrolases. Trends Endocrinol Metab 2011; 22: 218-25.

11 Lehner R, Cui Z, Vance DE. Subcellullar localization, developmental expression and characterization of a liver triacylglycerol hydrolase. Biochem J 1999; 338: 761-8.

12 Ko KW, Erickson B, Lehner R. Es-x/Ces1 prevents triacylglycerol accumulation in McArdle-RH7777 hepatocytes. Biochim Biophys Acta 2009; 1791: 1133-43.

13 Raison CL, Rutherford RE, Woolwine BJ, Shuo C, Schettler P, Drake $\mathrm{DF}$, et al. A randomized controlled trial of the tumor necrosis factor antagonist infliximab for treatment-resistant depression: the role of baseline inflammatory biomarkers. JAMA Psychiatry 2013; 70: 31 41.

14 Xiong J, Yang H, Wu L, Shang W, Shan E, Liu W, et al. Fluoxetine suppresses AMP-activated protein kinase signaling pathway to promote hepatic lipid accumulation in primary mouse hepatocytes. Int J Biochem Cell Biol 2014; 54: 236-44.

15 Feng XM, Xiong J, Qin H, Liu W, Chen RN, Shang W, et al. Fluoxetine induces hepatic lipid accumulation via both promotion of the SREBP1c-related lipogenesis and reduction of lipolysis in primary mouse hepatocytes. CNS Neurosci Ther 2012; 18: 974-80.

16 Xiao D, Chen YT, Yang D, Yan B. Age-related inducibility of carboxylesterases by the antiepileptic agent phenobarbital and implications in drug metabolism and lipid accumulation. Biochem Pharmacol 2012; 84: 232-9.

17 Du RH, Tan J, Sun XY, Lu M, Ding JH, Hu G. Fluoxetine inhibits NLRP3 inflammasome activation: implication in depression. Int $J$ Neuropsychopharmacol 2016; 19: pyw037.

18 Li K, Shen S, Ji YT, Li XY, Zhang LS, Wang XD. Melatonin augments the effects of fluoxetine on depression-like behavior and hippocampal
BDNF-TrkB signaling. Neurosci Bull 2017; 34: 303-11.

19 Su WJ, Peng W, Gong H, Liu YZ, Zhang Y, Lian YJ, et al. Antidiabetic drug glyburide modulates depressive-like behavior comorbid with insulin resistance. J Neuroinflammation 2017; 14: 210.

20 Masuda K, Nakanishi M, Okamoto K, Kawashima C, Oshita H, Inoue $\mathrm{A}$, et al. Different functioning of prefrontal cortex predicts treatment response after a selective serotonin reuptake inhibitor treatment in patients with major depression. J Affect Disord 2017; 214: 44-52.

21 Masand PS, Gupta S. Long-term side effects of newer-generation antidepressants: SSRIS, venlafaxine, nefazodone, bupropion, and mirtazapine. Ann Clin Psychiatry 2002; 14: 175-82.

22 Croft H, Settle E Jr, Houser T, Batey SR, Donahue RM, Ascher JA. A placebo-controlled comparison of the antidepressant efficacy and effects on sexual functioning of sustained-release bupropion and sertraline. Clin Ther 1999; 21: 643-58.

23 Wong DT, Perry KW, Bymaster FP. Case history: the discovery of fluoxetine hydrochloride (Prozac). Nat Rev Drug Discov 2005; 4: 764-74.

24 Farabaugh AH, Bitran S, Witte J, Alpert J, Chuzi S, Clain AJ, et al. Anxious depression and early changes in the HAMD-17 anxietysomatization factor items and antidepressant treatment outcome. Int Clin Psychopharmacol 2010; 25: 214-7.

25 Mallinckrodt CH, Goldstein DJ, Detke MJ, Lu Y, Watkin JG, Tran PV. Duloxetine: A new treatment for the emotional and physical symptoms of depression. Prim Care Companion J Clin Psychiatry 2003; 5: 1928 .

26 Raeder MB, Bjelland I, Emil Vollset S, Steen VM. Obesity, dyslipidemia, and diabetes with selective serotonin reuptake inhibitors: the Hordaland Health Study. J Clin Psychiatry 2006; 67: 1974-82.

27 Fjukstad KK, Engum A, Lydersen S, Dieset I, Steen NE, Andreassen $\mathrm{OA}$, et al. Metabolic abnormalities related to treatment with selective serotonin reuptake inhibitors in patients with schizophrenia or bipolar disorder. J Clin Psychopharmacol 2016; 36: 615-20.

28 Le Melledo JM, Mailo K, Lara N, Abadia MC, Gil L, Van Ameringen $\mathrm{M}$, et al. Paroxetine-induced increase in LDL cholesterol levels. J Psychopharmacol 2009; 23: 826-30.

29 Liebowitz MR, Gelenberg AJ, Munjack D. Venlafaxine extended release vs placebo and paroxetine in social anxiety disorder. Arch Gen Psychiatry 2005; 62: 190-8.

30 Grundy SM, Cleeman JI, Merz CN, Brewer HB Jr, Clark LT, Hunninghake $\mathrm{DB}$, et al. Implications of recent clinical trials for the National Cholesterol Education Program Adult Treatment Panel III Guidelines. J Am Coll Cardiol 2004; 44: 720-32.

31 Pedrinola F, Sztejnsznajd C, Lima N, Halpern A, Medeiros-Neto G. The addition of dexfenfluramine to fluoxetine in the treatment of obesity: a randomized clinical trial. Obes Res 1996; 4: 549-54.

32 Visser M, Seidell JC, Koppeschaar HP, Smits P. No specific effect of fluoxetine treatment on fasting glucose, insulin, lipid levels, and blood pressure in healthy men with abdominal obesity. Obes Res 1994; 2 : 152-9.

33 O'Kane M, Wiles PG, Wales JK. Fluoxetine in the treatment of obese type 2 diabetic patients. Diabet Med 1994; 11: 105-10.

34 Shahsavand Ananloo E, Ghaeli P, Kamkar MZ, Sadeghi M. Comparing the effects of fluoxetine and imipramine on total cholesterol, triglyceride, and weight in patients with major depression. Daru 2013; 21: 4.

35 Olguner Eker O, Ozsoy S, Eker B, Dogan H. Metabolic effects of antidepressant treatment. Noro Psikiyatr Ars 2017; 54: 49-56.

36 Kuehl LK, Muhtz C, Hinkelmann K, Dettenborn L, Wingenfeld K, Spitzer $\mathrm{C}$, et al. Association between major depression and cardiovascular risk: the role of antidepressant medication. Psychopharmacology 
(Berl) 2016; 233: 3289-95.

37 Kesim M, Tiryaki A, Kadioglu M, Muci E, Kalyoncu NI, Yaris E. The effects of sertraline on blood lipids, glucose, insulin and HBA1C levels: A prospective clinical trial on depressive patients. J Res Med Sci 2011; 16: 1525-31.
38 Fell MJ, Anjum N, Dickinson K, Marshall KM, Peltola LM, Vickers S, et al. The distinct effects of subchronic antipsychotic drug treatment on macronutrient selection, body weight, adiposity, and metabolism in female rats. Psychopharmacology (Berl) 2007; 194: 221-31. 